\title{
Simulation of X-ray Parameters via Thomson Scattering
}

\author{
S. H. Al-Shamma \\ M. I. Azawe \\ Department of Physic / College of Education \\ University of Mosul
}

Received

22 / 03 / 2010
Accepted

09 / 02 / 2011

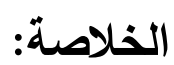

تم في هذا البحث تصميم برنامج حاسوبي لمحاكاة إستطارة ثومسون في توليد الأشعة

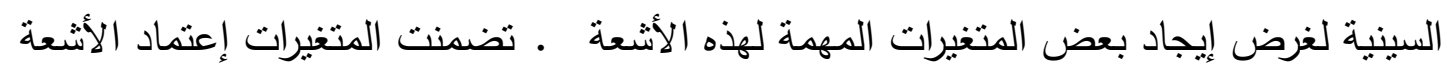

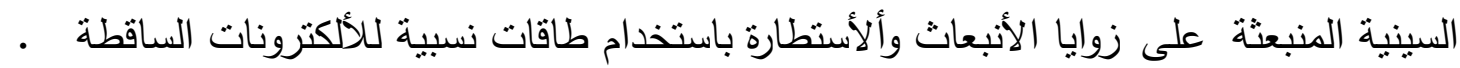

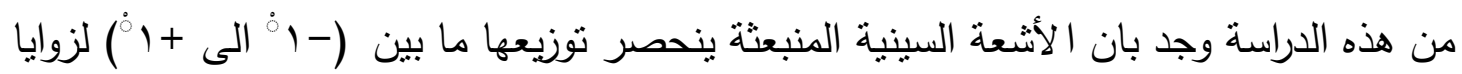

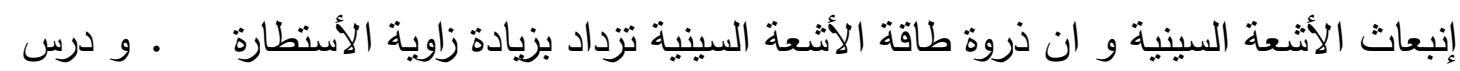

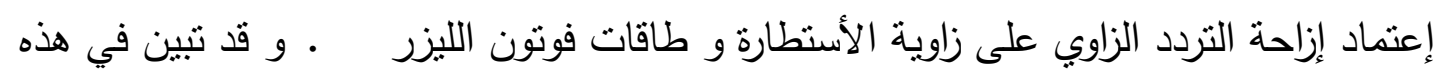

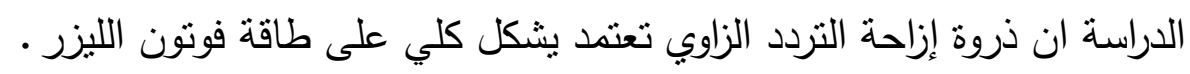

\section{ABSTRACT:}

In this work, a computer program has been built to simulate some important X-ray parameters via Thomson scattering. X-ray energy dependence on the emitting and $\mathrm{x}$-ray scattering angles for different incident electron energies were obtained. From this simulation, the $\mathrm{X}$-ray energy was found to be distributed around $\left(-1^{\circ}\right.$ to $\left.+1^{\circ}\right)$ for $\mathrm{X}$-ray emitting angle. Also it was found that, the X-ray peak energy was increased by increasing the scattering angles. An effort had been made on the angular frequency shift dependence on the scattering angle and laser photon energy. It had shown that the angular frequency shift was more dependent on laser photon energy rather than the scattering angle.

\section{1- Introduction:}

Thomson scattering is an important phenomenon via plasma physics and was first explained by physicist J.J. Thomson. Thomson scattering Xray based on the interaction between relativistic electron beam and high 
power laser pulses will provide a powerful tool for modern scientific research, due to its advantages over traditional light sources. Such as; good directional radiation, high brightness, wavelength tunability and very short pulse in the picoseconds and femtoseocnd region [1,2]. The generation of $\mathrm{x}$-ray radiation pulse have been attracted much attention since it could be a unique to investigate ultrafast dynamic process in nature such as the motion of electron in atoms $[1,2]$.

A short pulse X-ray sources is required in various research fields such as material, biology and medical science. The X-ray generation source via Thomson scattering using picoseconds and femtoseocnd laser light was developed and reviewed extensively by [2-9].

In this paper, a simulation had been made for Thomson scattering to obtain the X-ray energy dependence on the emitting and scattering angles for a wide range of different relativistic electron energies. Angular frequency shift depending on the scattering angle and laser photon energies were performed. The dependence of peak frequency shift on the incident electron energies was revealed.

\section{2- Theory:}

The generation of X-ray in the Thomson scattering (inverse of Compton scattering) can be obtained when a beam of relativistic electrons are collided with a laser light. The interaction geometry of Thomson scattering is shown in Fig.(1).

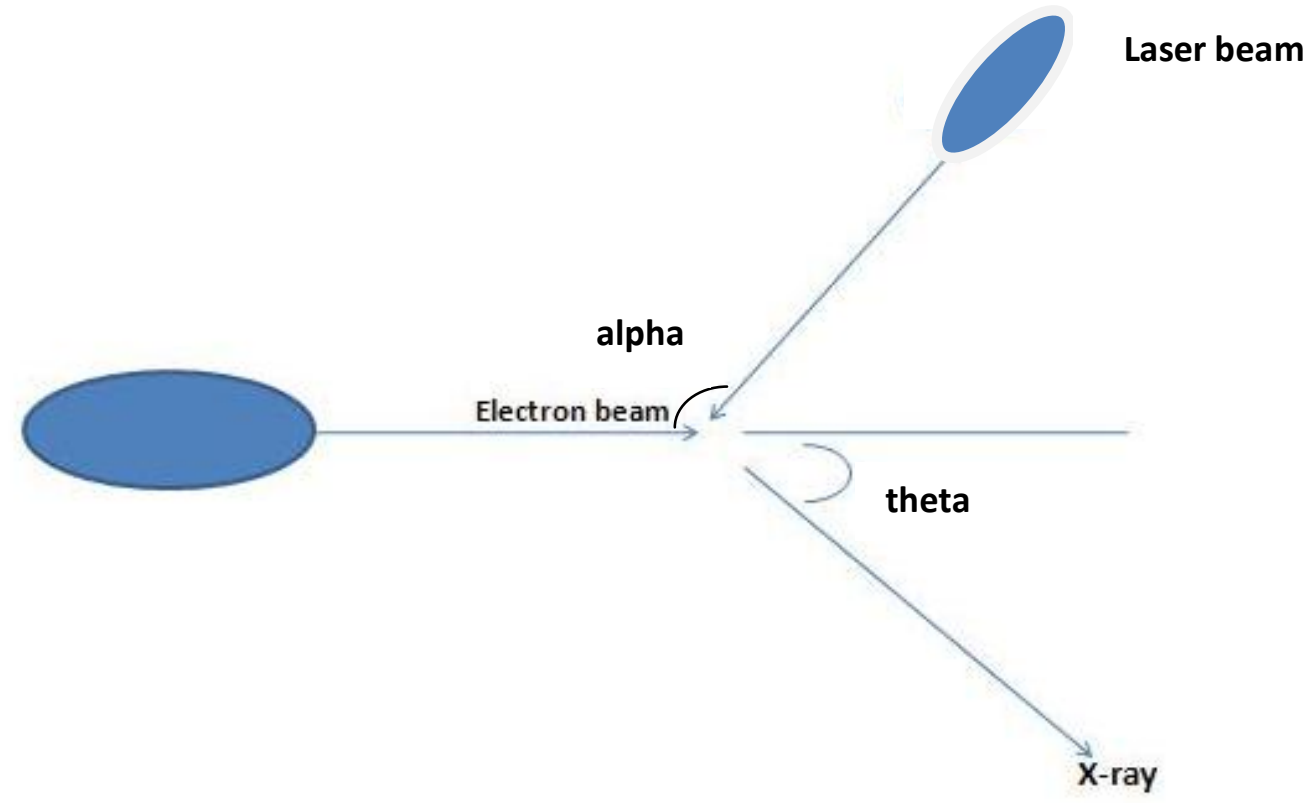

Fig.(1): Interaction geometry of Thomson scattering. 
The energy of the scattered X-ray is given by[4,6]:

$$
E_{x}=\frac{(1+B \cos \alpha)}{(1-B \cos \theta)} E_{\text {las }}
$$

Where $\boldsymbol{E}_{\text {las }}$ is the laser photon energy

$\boldsymbol{\theta}$ is the $\mathrm{X}$-ray emitting angle

$\boldsymbol{\alpha}$ is the scattering angle

B is the ratio of electron to light velocities $(\mathrm{v} / \mathrm{c})$.

The other expression of scattered $\mathrm{X}$-ray energy is given by[10]:

$$
E_{x}=\frac{2 \gamma^{2}}{1+\frac{a_{o}}{2}+\gamma^{2} \theta^{2}}(1-B \cos (\alpha))
$$

where $\boldsymbol{a}_{\boldsymbol{o}}$ is the normalized vector potential in the laser field and is given by:

$$
a_{o}=0.85 \sqrt{\left(I\left(W / c m^{2}+\lambda^{2}\right)\right)(\mu m)}
$$

where $\boldsymbol{\gamma}$ is the Lorentz factor of the electrons $\left(\gamma=\mathbf{1} /\left(\mathbf{1}-\left(\mathrm{v}^{2} / \mathbf{c}^{2}\right)\right)^{\mathbf{1 / 2}}\right)$

$\boldsymbol{\theta}$ is the angle of $\mathrm{X}$-ray observation

$\boldsymbol{\alpha}$ is the scattering angle.

Thomson scattering of relativistic electrons in the laser undulator field generate frequency up shifted radiation with the peak frequency given by[11]:

$$
w_{x}=\left[\frac{2 \gamma^{2}}{\left(1+\frac{a_{0}^{2}}{2}\right)}(1-\cos (\alpha))\right] w_{\square}
$$

where $\boldsymbol{w}_{\boldsymbol{o}}$ is the frequency of laser beam. Other parameters in the above equation were defined previously.

\section{3- Results and Discussion:}

Fig.(2) was obtained by using Eq.(2) for different relativistic electron energies such as $(100,60,40$ and $10 \mathrm{MeV})$ as shown in (a,b,c, and d) respectively. These figures were drawn in order to show the X-ray energy dependence on both X-ray emitting and scattering angles. It is obvious from these figures that the maximum $\mathrm{X}$-ray energy was around $\left(-1^{\circ}\right.$ to $\left.+1^{\circ}\right)$ of the -ray emitting angles. On the other hand, the X-ray peak energy increased by increasing scattering angles. Furthermore, the X-ray energy reached its maximum values $\left(4 \times 10^{4}, 1.5 \times 10^{4}\right.$, and $\left.0.1 \times 10^{4} \mathrm{eV}\right)$ for the incident electron energy of $(100,60,40$, and $10 \mathrm{MeV})$ respectively. 
Simulation of X-ray Parameters via Thomson Scattering.
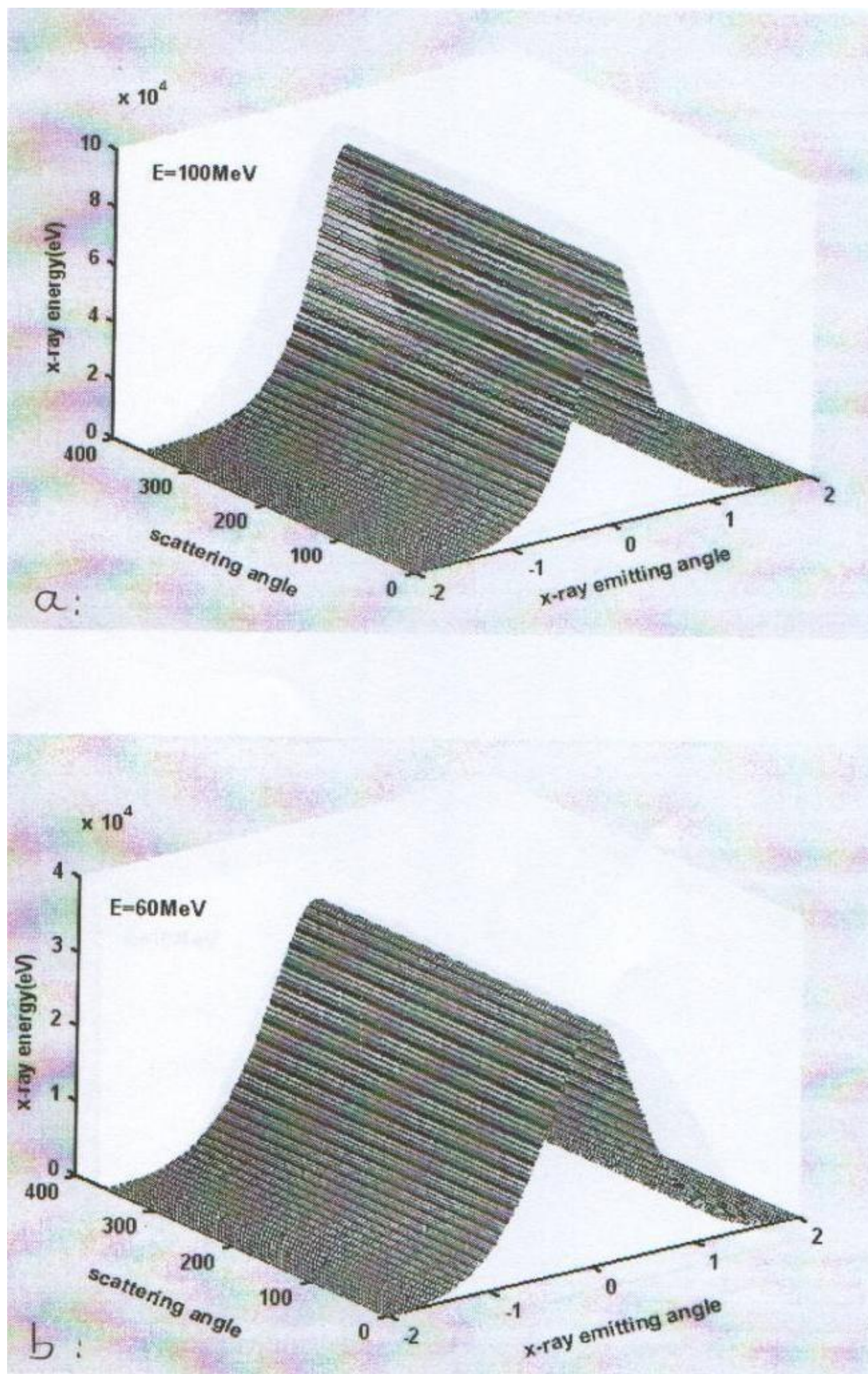

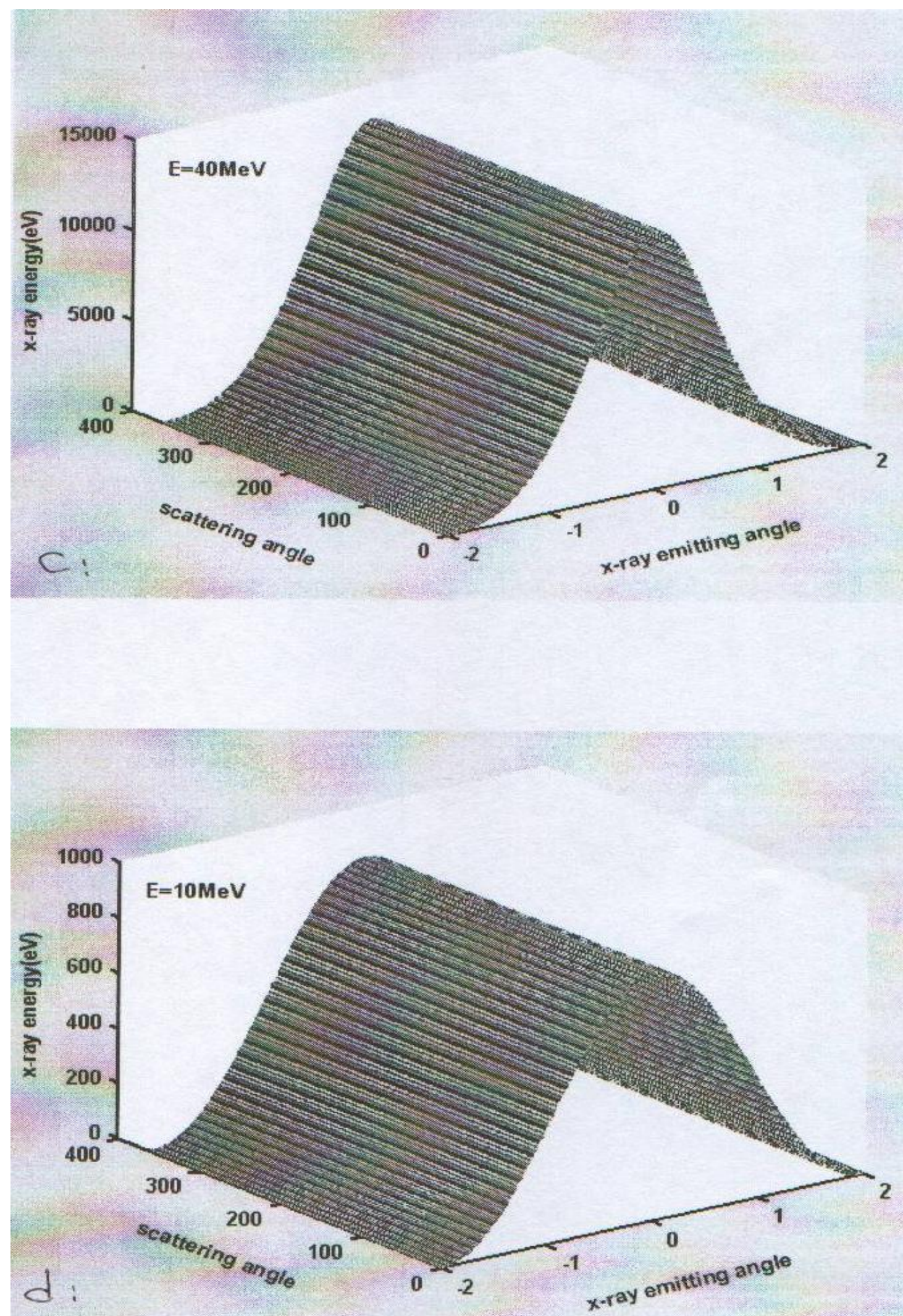

ig (2) Dependence of the x-ray on the emitting and scattering angles for different incident electron energies as in (a,b,c and d) 
In order to study the X-ray energy taking into account the laser light intensity and wavelength, as given by Eq.(3), the simulation was carried out for this purpose and the results were shown in Fig.(3). It is evident, on comparison with Fig.(2), that the peak energy with the incident electron energy as in (a,b,c, d,e and f). In addition to that, the periodicity was appeared starting from the incident electron energy equal to $(20 \mathrm{MeV})$ as shown in Fig.(3d).
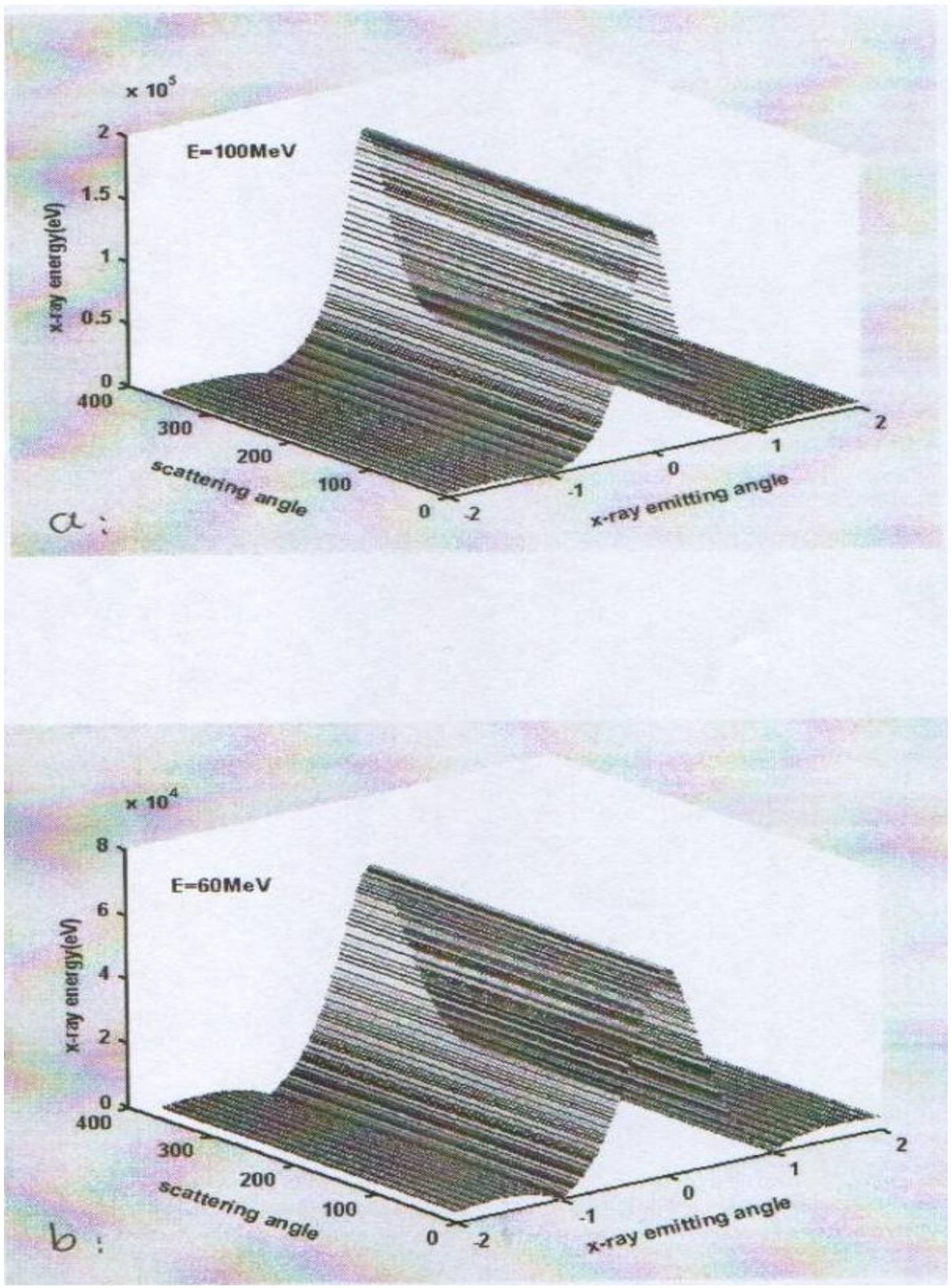

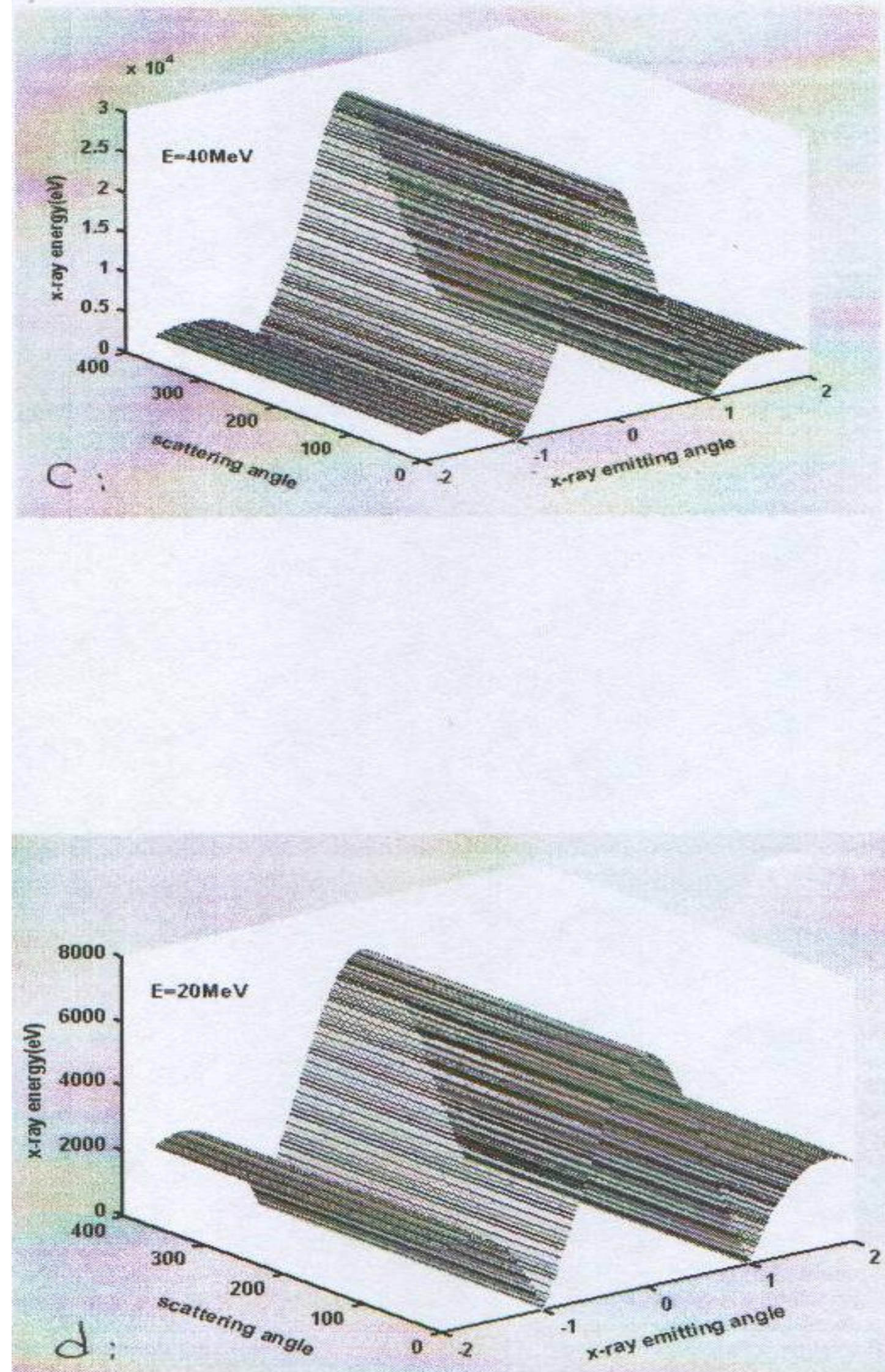
Simulation of X-ray Parameters via Thomson Scattering.
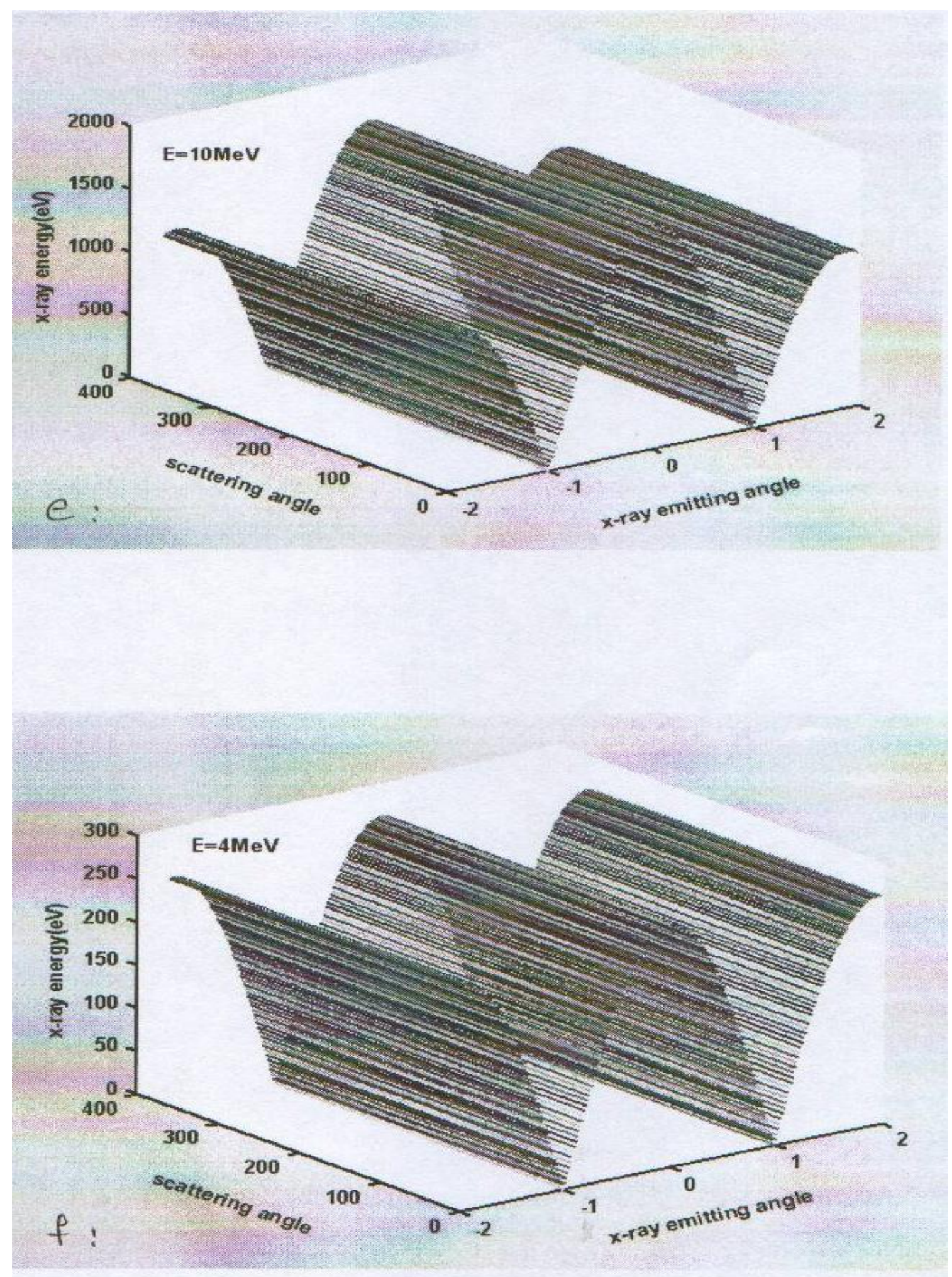

g (3) Dependence of the x-ray energy on the emitting and scatteri for different incident anergies as in (a,b,c,d,e and $\mathrm{f}$ ) 
Fig.(4) illustrates the angular frequency shift as a function of scattering energy and laser photon energy for different incident electron energies $(80$ and $10 \mathrm{MeV})$. In fact, one can deduce from the diagrams that the angular frequency shift was more dependent on the laser photon energy while the peak frequency shift was dependent on the scattering angle.
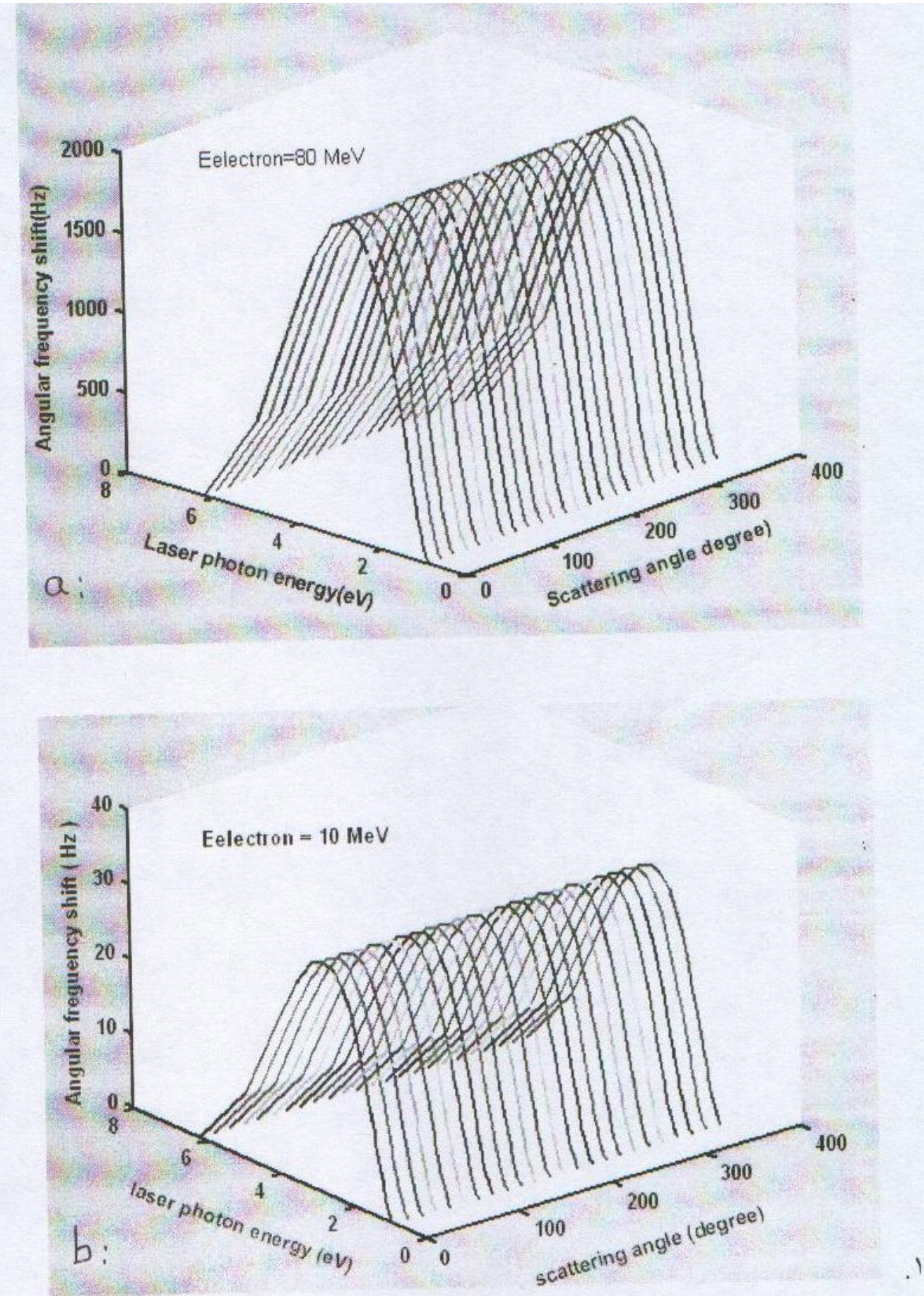

Fig (4) 3D plots of the angular frequency shift as a function of the scattering angle and photon energy for different incident electron energies as in ( $\mathrm{a}$ and $\mathrm{b}$ ) 
Fig.(5) shows the dependence of the peak frequency as a function of incident electron energy. Nonlinearity can be seen from the graph for this dependence, which suggests how X-ray parameters can be altered with the incident electron energy in addition to the laser energy and wavelength.

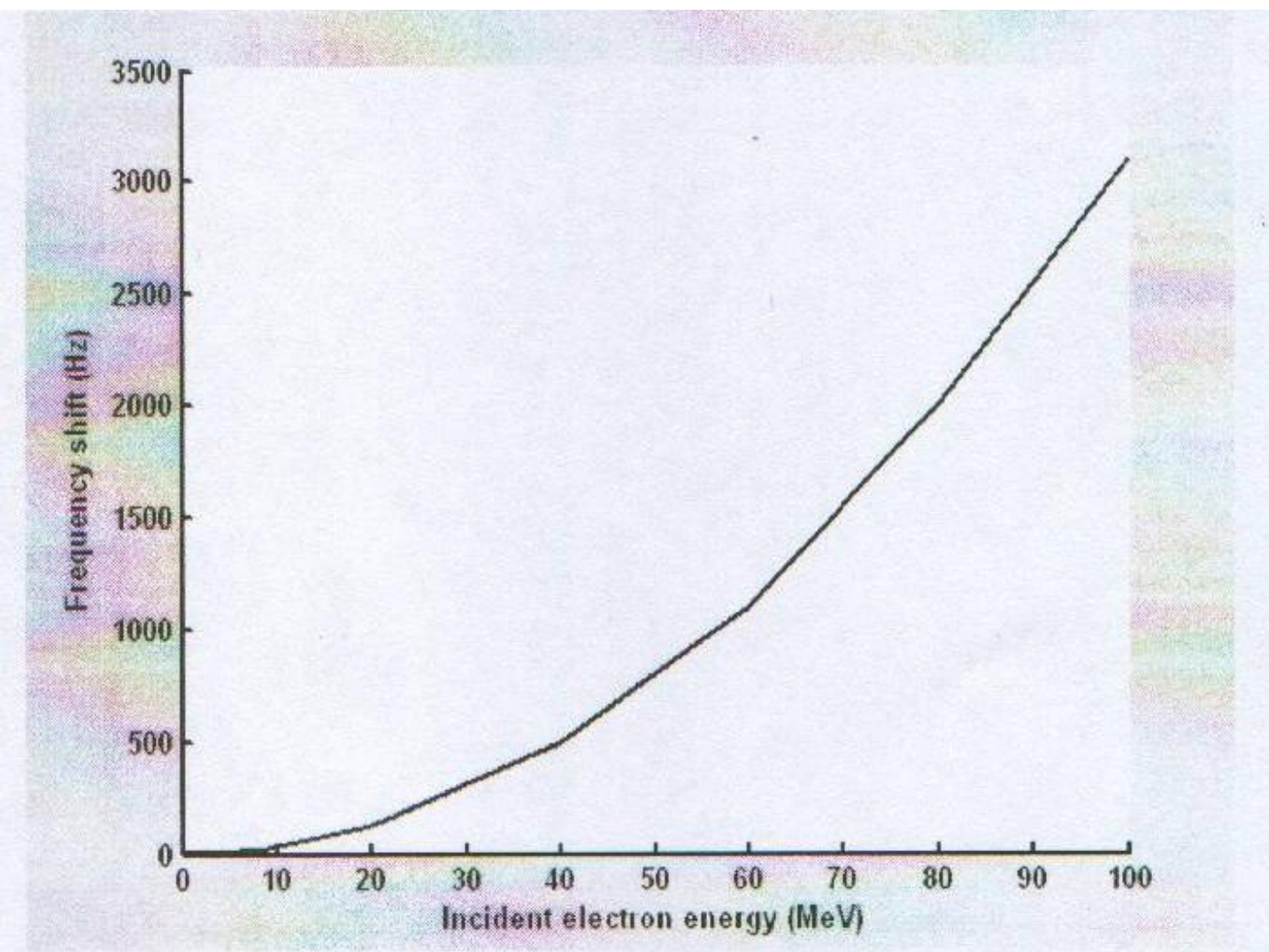

Fig (5) peak frequency shift as a function of incident electron energy

\section{4- Conclusions:}

From this simulation, we can conclude that the $\mathrm{X}$-ray energy is like a bell-shape dependence mainly on the X-ray emitting angle. But the peak values of $\mathrm{X}$-ray energy is scattering angle dependence. A periodicity was observed in the behavior when both laser intensity and wavelength were included in the X-ray scattering equation. Finally, the angular frequency shift was increased exponentially with the incident electron energy. 


\section{References}

1) www.Sunysb.edu/icfa2001/papers/w2.5-pdf

2) W. H. Huang, W. H. Huang, H. B. Chen, C. Chheng, Y. Cheng, Q. Du, T. B. Du, Y. CH. Du, X. Z. He, J. F. Hua, G. Huang, Y. CH. Ge, Y. ZH. Lin, B. Xia, M. Jxu, X. D. Yuan, SH. X. Zheng. Procceedings of the 2004 FEL conf.,622-624, Trieste, Italy.

3) J. Yang, M. Y. Yorozu, F. Sakai, T. Yangida, Y. Okada, T. Nakajyo. Proc. of EPAC 2002, Paris, France.

4) A. Zelinsky, E. Bulyak, P. Gladkikh, I. Karnaukhov, A. MProc. of EPAC 2004, Lucerne, Switzerland.

5) F. Saka, J. Yang, M. Yorozu, Y. Okada, T. Yanagida Proc. of the 2001 Particle Accelerator Conf., Chicago, USA.

6) www.thales-laser.com

7) Y. Lin-Xin, Chinese Phys. C 33,154-156, 2009.

8) Fumio Sakai, Jinfeng Yang, Masafumi Yorozu, Yasuhiro Okada, M. Washio. proceeding of 2004 FEL conference, 466, Trieste, Italy.

9) R. Kuroda, D. Ueyama, T. Saito, S. Minamiguchi, K. Hidume, M. Washio. proceeding of 2004 FEL conference, 466-469, Trieste, Italy.

10) GPriebeD. laundy, M. A. Masdonald, G. P. Diakun, S. P. Jamison, P. J. Phillips, B. Shechy, L. B. Jones, D. J. Holder, S. L. Smith, G. J. Hirst, N. Naumova, G. A. KRafft, G. A. Krafft, K. Spohr, H. Legall, J. B. Rosenzweig, F. Grunger, C. P. J. Barty, C. Siders, U. Schramm, S. Chattopadpadhyay and E. A. Seddon. SPIE Conf. soft X-ray laser and applications VII, San Diego, USA, 2008.

11) K. Nakajima, H. Nakanishi, A. Ogata.1998, IEEE. 